\title{
APROXIMACION AL SISTEMA IMPOSITIVO DE LA GENERALIDAD: EL TALL DEL DRAP EN EL AREA ALICANTINA (SIGLOS XIV Y XV)
}

\author{
Maria Rosa Muñoz Pomer \\ Universidad de Valencia
}

\section{INTRODUCCION}

La clasificación de los impuestos puede hacerse, al menos, desde dos puntos de vista: en base a la titularidad o al concepto. La viabilidad de la primera ha sido puesta en crisis por algunos autores que se inclinan por la segunda (1). La utilidad de esta última es, a nuestro juicio, indudable, pero requiere, en el confuso panorama de los impuestos medievales valencianos, una definición previa de los conceptos impositivos que permita la mencionada clasificación.

Aquí intentamos, siguiendo un primer esquema presentado en la 1.Assemblea d'Historia de la Ribera (1980) (2), compatibilizar ambas.

Tres fueron los titulares que establecimos en aquella ocasión: el rey, el municipio y el General. A tenor de ella, y sin entrar en cuestionar la titularidad de los poseedores de señoríos, vamos a centrar nuestra atención en los impuestos dependientes del último: la Generalidad, escasamente explicitados en aquella ocasión.

La fuente financiera principal de la Generalidad, surgida de las Cortes del trescientos (3), son los impuestos que clasificados conceptualmente se dividen en directos e indirectos. Ninguno de los dos admite una segunda subdivisión en ordinarios y extraordinarios. Los primeros, sin embargo, habría que situarlos entre estos últimos, y se conocen con el nombre fogatge; los segundos, designados por las cortes de 1362-63 generalidades, pueden situarse desde 1404 entre los ordinarios. 
Estas, definidas por Camarena como «impuesto sobre la entrada y salida de mercancias en el Reino o sobre su compra y consumo en el interior" (4), abarcan distintos productos que son agrupados según el mismo autor en once capítulos. Aquí no podemos hacernos eco de todos ellos por lo que hemos centrado nuestra atención en uno de los más significativos por el volumen que alcanza en el total de ingresos, el tall del drap (5). Aunque el ámbito territorial de este impuesto abarque, tal como quedará explicitado en el análisis de sus elementos, todo el reino, nuestro estudio se dirigirá preferencialmente al área alicantina.

\section{ORIGEN Y EVOLUCION DEL IMPUESTO}

Los fueros recogen en ocasiones legislación sobre impuestos, no es fácil, sin embargo, encontrar allí las disposiciones y normas de muchos de ellos surgidos para adecuar la realidad financiera a los retos que plantea la sociedad circundante.

Este es el caso de las generalidades, arbitradas por las Cortes para hacer frente a los múltiples problemas a los que se enfrentará la corona en los siglos XIV y XV. Su origen, pues, hay que buscarlo en los acuerdos de aquellos.

La primera documentación, relativa a un impuesto general extraordinario acordado en Cortes que ha llegado hasta nosotros, procede de las celebradas en Valencia en 1329. Los elementos obligados en aquella ocasión nada tienen que ver con el tema que ahora nos ocupa (6).

Las Cortes de Valencia de 1342-1343 introducen el gravamen sobre los tejidos, además de los productos cargados en las anteriores: cereales, harina, carne y vino. La documentación consultada no aporta otras referencias que posibiliten la comparación de los elementos de ambos impuestos para establecer su dependencia.

Las Cortes de 1362-1363 crean una serie de impuestos para hacer frente al donativo prometido al rey. Estos son bautizados por la misma asamblea con el nombre bajo el que serán conocidos en el futuro: generalitats, e inciden en actividades como la fabricación, venta y exportación de ciertos tejidos. Su base imponible y liquidable se establece sobre el precio alcanzado por los mismos. El tipo de gravamen queda fijado en un sueldo por libra del total del precio.

La conexión de este impuesto y el creado en 1404 es evidente, tal y como quedará demostrado en el análisis de sus elementos. Se puede hablar, sin duda, más de adaptación que de creación en este último caso. Dado el primer paso, las necesidades surgidas a raíz de la guerra de "los dos Pedros" actuaron como factor de cambio de las estructuras físcales consolidando los impuestos dependientes del General.

Las Cortes de Cullera-Valencia de 1364 y las de Sagunto de 1365 vuelven a contemplar este concepto impositivo, aunque no añaden nada a 
lo ya establecido. Las de 1367 utilizan el término imposicions en lugar de generalitats; los tejidos, incorporados años antes, no dejan tampoco de figurar aquí, aunque en este caso incide sobre la exportación.

Las Cortes de San Mateo-Valencia de 1369-1370 recuperan el término. Ahora se grava la venta de tejidos de lana, estambre, lino, cáñamo o estopa, para la confección y la exportación de los mismos. Se acuerda en esta asamblea la exención de los tejidos donados a los pobres o a la iglesia.

El final de la mencionada contienda no supone disminución en las necesidades de la hacienda real. La política mediterránea de Pedro IV se encargará de absorber las cantidades que, como en otras ocasiones, deben procurar las Cortes.

Reunidos en Valencia en 1371 con esa finalidad, proporcionar fondos, recurren nuevamente a las generalidades. La documentación consultada no permite, en este caso, explicitar los elementos de las mismas, pero no es arriesgado suponer la vinculación a los acuerdos de reuniones anteriores, forma que se adoptará en las celebradas con posterioridad: Cortes de 1373-1374.

Las generales reunidas en Monzón (1375-1376) con el fin de atajar las pretensiones del duque de Anjou sobre Mallorca, Rosellón y Cerdeña, recurren nuevamente a ellas, al igual que se hará en las de 1382-1383 para recaudar el adelanto prometido al monarca.

Los primeros contratos de arrendamiento, que hemos localizado en los protocolos notariales, pertenecen a 1384 e inciden sobre la exportación aunque el que dedica a la sal grava también el consumo. El resto se agrupa en tres capítulos: tejidos de oro, seda, camelote, lana y estambre para el comercio; lanas, añinos, tejidos de lana y estambre sin terminar e hilazas de ambos materiales; tintes, fibras vegetales, especias, cerámica y otros productos. El del tall o tejidos vendidos para la confección no ha sido hallado, aunque se menciona por exclusión en el de la exportación de tejidos: «En axí, que en lo dit capítol no son ne sien enteses... draps apparellats a obs de tayll com ja paguen en altres capitols". No nos es posible, por tanto, ofrecer sus elementos.

1390 es la fecha en que volvemos a encontrar contratos de arrendamientos de las generalidades. La adopción de las mismas responde a la decisión de los dieciocho miembros, encargados por el rey para resolver la oferta de las Cortes de 1388-1389, que recurren a los procedimientos habituales: compartiment y generalitats.

Aquí nos encontramos ya con contratos similares a los de 1404 . El hecho de que el organismo no disponga todavía de la infraestructura administrativa adecuada dificulta la localización de la documentación, donde se recoge su actividad, encargada a distintos notarios:

Los contratos localizados, relativos al Tall del drap, comprenden una zona más o menos amplia que abarca una o varias localidades. Todas 
ellas situadas en territorio castellonense o valenciano. Su existencia para Alicante viene confirmada, sin embargo, por las épocas extendidas por los clavarios (7) donde se reconocen algunas cantidades cobradas por el arrendamiento del Tall del drap de Alicante, Cocentaina, Elche, Orihuela para 1391.

El examen de sus lementos lo hemos efectuado a través de los conservados, ya que todos ellos establecen las mismas condiciones en relación al impuesto.

Comparando éstas con las establecidas en 1404 se aprecian escasas diferencias: cambio de lugar de alguna cláusula, falta de otras (8) y mayor brevedad en algún caso (9). Sólo varía la tarifa del impuesto que en esta ocasión queda fijada en 6 dineros por libra de precio.

\section{RECAUDACION DEL IMPUESTO}

Los responsables de los impuestos, nominados por la misma asamblea, son los encargados de la gestión del mismo. ¿Cuál fue la fórmula adoptada para su recaudación? Nos inclinamos por el arrendamiento al mayor postor, método habitualmente empleado en la época por las haciendas real y municipal.

El primer contrato localizado es, como ya expusimos, de 1384, el último de 1441. La localización de las generalidades se hace en subasta pública al mayor postor. La venta, en ocasiones, se sostiene o anima desde la misma institución a través de seguros y pujas (10).

Los diputados, realizado el encante y mientras el notario extiende el documento público al comprador, envían misivas a los oficiales y responsables de cada lugar para que, entre tanto, se respete su derecho.

Los contratos de arrendamientos proporcionan los elementos del impuesto, los individuos que se hacen responsables de su cobro y la cantidad que han ofrecido. Esta cifra es global y sólo de forma indirecta se puede relacionar con los ingresos. No permiten, pues, aproximarnos al comercio, fabricación y consumo. Sería posible a través de ellos, sin embargo, fijar el volumen de rentas que el Tall alicantino supone en el total que por este concepto recibe la Generalidad, pero tampoco ello es factible para todo el periodo 1391-1443. Sólo disponemos de los contratos de 1404, los anteriores se encuentran dispersos en los protocolos notariales, y a partir de 1405 el Tall sufre una reestructuración quedando dividido en tres bloques: el Tall de Valencia, el de Orihuela y el del reino.

El arrendamiento global de este último a un solo postor impide el propósito anunciado, ya que los subarriendos realizados plantean el mismo problema: su localización (11).

Nos limitamos pues a ofrecer los de 1404 con carácter meramente indicativo. 


\begin{tabular}{lr}
\hline \multicolumn{1}{c}{ Quarter } & Precio \\
\hline 1. Alcira, etc. & $5.200 \mathrm{~s}$. \\
2. Alpuente, etc. & $2.000 \mathrm{~s}$. \\
3. Ayora, etc. & $450 \mathrm{~s}$. \\
4. Burriana, etc. & $2.400 \mathrm{~s}$. \\
5. Cocentaina, etc. (13) & $3.300 \mathrm{~s}$. \\
6. Cullera, etc. & $1.300 \mathrm{~s}$. \\
7. Gandia, etc. (14) & $7.000 \mathrm{~s}$. \\
8. Játiva, etc. & $12.700 \mathrm{~s}$. \\
9. Liria, etc. & $1.650 \mathrm{~s}$. \\
10. Onda, etc. & $3.000 \mathrm{~s}$. \\
11. Onteniente, etc. (15) & $1.500 \mathrm{~s}$. \\
12. Orihuela, etc. (16) & $2.000 \mathrm{~s}$. \\
13. Sagunto, etc. & $3.300 \mathrm{~s}$. \\
14. San Mateo, etc. & $5.500 \mathrm{~s}$. \\
15. Segorbe, etc. & $8.200 \mathrm{~s}$. \\
\hline Suma total & $59.500 \mathrm{~s}$. \\
\hline
\end{tabular}

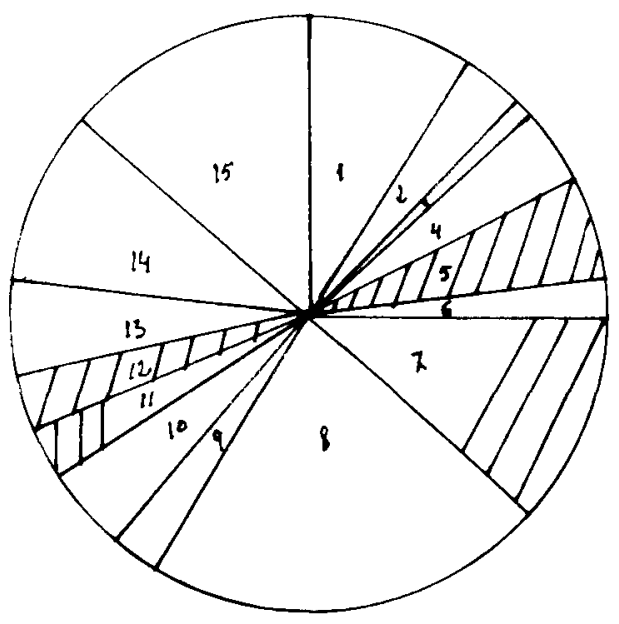


Si atendemos a los datos expresados en el cuadro anterior la suma que aporta el territorio alicantino a la Generalidad por este concepto es inferior al 23 ' $19 \%$ del total. Hay que tener en cuenta que hemos sumado los precios que alcanzan los quarters: $5,7,11$ y 12 y algunos de ellos comprenden lugares ajenos al término provincial actual de Alicante.

La que representa a su vez el tall, en el conjunto de las generalidades, queda claramente evidenciado en la representación gráfica elaborada a partir de los precios alcanzados en la venta de las mismas ese año.

\section{ARRENDAMIENTO DE LAS GENERALIDADES EN 1404 (17) SU REPRESENTACION GRAFICA}

\begin{tabular}{ll}
\hline \multicolumn{1}{c}{ Generalidades } & Precio \\
\hline 1. Cuero y vellones & $20.050 \mathrm{~s}$. \\
2. Lanas & $50.000 \mathrm{~s}$ \\
3. Madera y vino & $18.020 \mathrm{~s}$. \\
4. Mercaderias & $20.050 \mathrm{~s}$. \\
5. Pieles y arroz & $12.000 \mathrm{~s}$. \\
6. Sal & $40.000 \mathrm{~s}$ \\
7. Tall de Orihuela & $2.000 \mathrm{~s}$. \\
8. Tall de Valencia & $90.100 \mathrm{~s}$. \\
9. Tall del reino & $57.500 \mathrm{~s}$. \\
10. Tinteós & $35.000 \mathrm{~s}$. \\
\hline Suma total & $344.720 \mathrm{~s}$. \\
\hline
\end{tabular}

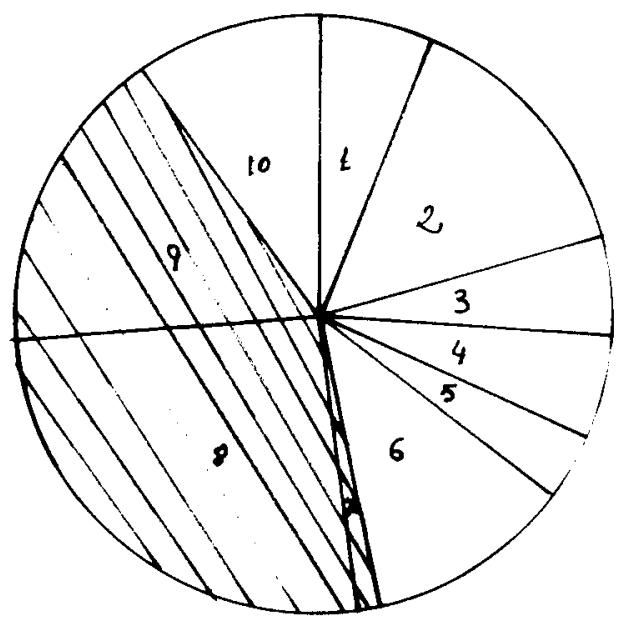


Se observa que el tall dividido en tres capitulos: el de Orihuela, Valencia y el reino es el que más volumen de ingresos representa en relación al resto, aunque no alcanza a la mitad de entradas por este concepto. El de Valencia es, sin duda, el que produce mayores ingresos y representa el $26{ }^{\prime} 13 \%$ en relación al total de sueldos obtenidos por generalidades.

La redacción de la documentación, tal y como puede observarse en las notas 14,15 y 16 y las noticias de 1391 nos inclinan a pensar que los cuarteles o quarters fueron más reducidos. A partir de la documentación de 1404 hemos efectuado la siguiente subdivisión de los mismos (18). Ese territorio se fracciona a su vez en partes más pequeñas denominadas filloles, cuya extensión no ha sido posible determinar.

\section{QUARTER POBLACIONES QUE COMPRENDE}

\begin{tabular}{|c|c|}
\hline $\begin{array}{l}\text { Alcoy } \\
\text { Alicante }\end{array}$ & Penáguila, Ibi, Castalla, Tibi, Jijona. \\
\hline Cocentaina & Planes. \\
\hline Denia & $\begin{array}{l}\text { Pego, Ondara, Vall de Laguart, Murla, Jávea, Castell de } \\
\text { Castell, Tarbena, Confrides, Guadalest, Callosa, Calpe, } \\
\text { Altea, Polop, Relleu, Finestrat, Benidorm y Villajoyosa. }\end{array}$ \\
\hline Elche & $\begin{array}{l}\text { Petrel, Elda, Monóvar, Pinoso, Novelda, Aspe y Crevi- } \\
\text { llente. }\end{array}$ \\
\hline Gandía (19) & Palma, Villalonga, Gallinera, Ebo y Alcalá. \\
\hline Onteniente & Albaida, Bocairente, Agres, Bañeres y Biar. \\
\hline Orihuela & Guardamar. \\
\hline
\end{tabular}

El cuartel que abarca Orihuela y Guardamar es el único, al arrendarse independientemente del general del tall del reino, que nos ha legado el valor anual. El hecho de que lo arriende el consejo de Orihuela influye, sin duda, en el precio, el cual, si al principio oscila, 2.000 sueldos en 1404 y 1.300 en 1406, a partir de 1407 y hasta 1424 se mantiene constante en 2.500 sueldos. En 1425, como ocurrió en 1405, se arrienda con el total del reino.

La venta conjunta del resto de quarters del reino en un sólo contrato a partir de 1405 facilita la gestión del impuesto, al eliminar arrendatarios. Los subarriendos aunque son comunicados por los diputados a las autoridades y habitantes oportunos, no dependen económicamente de la institución. 


\section{EL CONTRATO (20)}

El documento firmado entre los contratantes, los diputados y el arrendatario, consta de dos partes claramente diferenciadas. Una, en la que constan las condciones particulares y generales del impuesto, dividida en varias cláusulas que se repite anualmente con ligeras variantes y que son las que se toman como base para la subasta. Otra, donde se estipula: la fecha de venta, procedimiento empleado para la misma, tiempo y precio alcanzado, así como su adjudicación. Figuran también los fiadores y los testigos que presenciaron el acto. Se recogen en ocasiones otro tipo de anotaciones como las cantidades prometidas a los licitadores de la subasta y la fecha en que ha sido clausurado el contrato.

\subsection{Cláusulas comunes a otras generalidades}

Los contratos son anuales hasta 1439. Los firmados en 1441 tendrán una validez de tres años. El precio del mismo debe satisfacerlo el comprador en seis pagas iguales a abonar cada dos meses al clavario del brazo real encargado de la contabilidad de la Generalidad. Los compradores, cuyo número no podrá exceder a seis, deben ofrecer las oportunas fianzas antes de proceder al cobro del impuesto.

Las cuestiones que surjan, entre los arrendatarios y los que deben pagar o los que han cometido fraude, quedan bajo la jurisdicción de los administradores, sus delegados o los jurados del lugar si faltan los primeros. El proceso se realizará de palabra y la sentencia no podrá ser recurrida ante ningún otro juez.

Las dudas referentes a las cláusulas de este impuesto serán también resueltas verbalmente por los administradores de los mismos que son tres: uno por cada brazo que tienen representación en Cortes.

El comprador del impuesto gozará de un plazo de seis meses, después de acabado el tiempo del arrendamiento, para cobrar todas las deudas por ese concepto, pasado ese término perderá todo derecho sobre aquellas.

Los recaudadores, que cobren sobre lo que no deben a más cantidad de la establecida, serán sancionados con una multa de 60 sueldos y obligados a devolver el doble de lo obtenido.

Las vicisitudes favorables o desfavorables en torno al cobro corren a cargo de los arrendantes, los diputados se retienen el derecho a aumentar o disminuir los artículos sometidos y el valor del impuesto.

\subsection{Elementos del impuesto}

Estos han sido extraídos de los capítulos subastados en 1405 . EI amplio periodo de vigencia de este impuesto (siglo XV al XVIII) sin duda 
introducirá variantes en algunos de sus elementos. Aquí sólo quedan reseñadas las alteraciones producidas hasta 1440-1443, fecha de los últimos contratos de arrendamiento que se conservan.

\subsubsection{Actividades sujetas y exentas}

\section{Actividades sujetas}

- La venta de tejidos de oro, seda, camelote, lana (21), estambre, lino, algodón, cáñamo, estopa, ginesta o de otra clase para confección (22).

- La fabricación casera de los mismos para la venta.

- La importación-exportación con el mismo fin.

- Importación de túnicas, cotas, capirotes, calzas y otras piezas nuevas para vender (23).

Actividades exentas

- Las telas donadas a los pobres o a la Iglesia.

- Los paños de lino o estopa hechos en casa para el consumo de sus moradores.

- La transformación de piezas viejas hechas por los ropaveje$\operatorname{ros}(24)$.

- Los tejidos vendidos para uso del santo padre, cardenales, camarlengo y sus familiares (25).

\section{Ambito temporal y territorial del impuesto}

Establecido con carácter extraordinario, los acontecimientos políticos favorecen su consolidación a partir de 1404 y hasta el siglo XVIII.

El territorio se divide para su cobro en quarters y filloles. Los primeros, bautizados con el primer topónimo que se relaciona en los arrendamientos, suman en total 27 demarcaciones; seis de ellas abarcan la provincia de Alicante si exceptuamos Agres, Bañeres y Biar que se incluyen en el de Onteniente y Gallinera, Alcalá y Ebo que se incluyen en el de Gandía según se recoge en el apartado 3 .

\subsubsection{Sujeto Pasivo}

\section{Contribuyentes}

- Los vendedores de tela.

- Los que tejen en casa para la venta. 
- Los importadores o exportadores de paños con el mismo fin, incluidos los ropavejeros.

- Los importadores de ropa.

Personas exentas

- Los que fabrican para su propio consumo.

Repercusión del impuesto

Los contribuyentes hacen recaer el peso del impuesto en los consumidores. Incluso el rey queda sometido al mismo.

\subsubsection{Base del impuesto}

Base imponible

El impuesto se establece en base al precio que alcanzan las telas y ropas.

Régimen para la determinación de la base

La determinación corresponde a los compradores o recaudadores del impuesto que sellan el paño para evitar fraudes.

\subsubsection{Deuda tributaria}

Tipos de gravamen

Todas las operaciones sometidas a este impuesto quedan gravadas por un sueldo por libra de precio (26).

\section{Base liquidable}

Coincide con la imponible ya que la documentación no recoge ninguna deducción aplicable.

Pago

- El fabricante o vendedor de paños no puede venderlo hasta que no haya satisfecho el impuesto y la tela haya sido sellada.

- Los que lo hacen en casa para la venta o los importan deben manifestarlo a los compradores y recaudadores del impuesto. 
- Todos los que venden telas para confección quedan obligados a prestar juramento, sólo una vez y a requerimiento de los responsables del cobro de la imposición, de que han satisfecho las cantidades debidas por este concepto.

- Las prendas de vestir confeccionadas, independientemente de donde se hicieran, en el quarter donde se venden.

\section{Sanciones}

- El vendedor que despache tejidos sin selio, perderá la tela cada vez que contravenga el impuesto.

- Los sastres y tundidores que trabajen géneros sin sello quedan sometidos a 60 sueldos de sanción cada vez. El dueño del mismo debe abonar, además, la generalidad y si es ropavejero el doble.

- Los que introducen telas o las hacen en casa para su venta y no las declaran, tal como quedó establecido, quedan sometidos a la pena de 20 sueldos y al pago duplicado de la generalidad.

- Los vendedores y ropavejeros que hayan prestado juramento en falso sobre el debido pago del impuesto quedan obligados a pagar una sanción de 60 sueldos y a pagar el doble de lo que debian satisfacer por generalidad.

- Todo ropavejero que compre telas debe manifestarlo al comprador o recaudador del impuesto y declarar bajo juramento cuál es el uso para el que lo adquirió: corte o como mercancía, en caso contrario será sancionado con seis libras.

Esa misma sanción recaerá en los corredores a los que se les encomienden géneros para vender y no los declaren en el lugar donde se cobra el impuesto.

- Para evitar los fraudes los administradores autorizan el registro en casa de los sastres y tundidores aunque con ciertas condiciones (28).

El dinero recaudado por las sanciones se divide en tres partes: una para el General, otra para el manteniemiento de las fortificaciones del lugar donde se cometió el fraude, o el común de aquella, la tercera parte para el acusador.

Las sanciones pueden ser rebajadas por los administradores de las generalidades, pero no anuladas.

\section{DEFINICION Y CLASIFICACION}

Este impuesto, de origen extraordinario, se convierte de hecho en ordinario a partir de 1404 aunque el organismo no se consolida hasta 1418. Según el análisis de sus elementos se le puede calificar de indirecto, 
ya que grava una actividad y su recaudación depende de aquella, independientemente del sujeto que la realiza.

Los impuestos cuya titularidad corresponde a la Generalidad se dividen, tal como expusimos, en extraordinarios y ordinarios. Los primeros, temporales, arbitrados para sufragar parte del donativo ofrecido por las Cortes se recaudan en base a los hogares ofochs. Los segundos, entre los que se encuentra el tall, son más numerosos y proporcionan mayores ingresos.

El análisis de la documentación consultada para este trabajo nos permite avanzar aquí la clasificación de los impuestos dependientes de la Generalidad, aunque los elementos de todos ellos, excluido el del tall, todavía no hayan sido debidamente normalizados. Aplicamos el doble sistema anunciado en la introducción: la titularidad y el concepto.

Las actividades gravadas son importación-exportación y la venta por consumo en el reino. La documentación las agrupa por afinidades de productos. Algunos de ellos cotizan por los dos conceptos. Nuestra clasificación responde, como puede observarse, a la actividad, en ella se han incluido los productos que deben abonar este impuesto.

\section{IMPUESTOS DEPENDIENTES DE LA GENERALIDAD EN LA PRIMERA MITAD \\ DEL SIGLO XV}

DIRECTOS (extraordinarios): fogatge. Su tipo o tarifa oscila de unas Cortes a otras.

INDIRECTOS (ordinarios): Generalidades. Su tipo, generalmente sobre el precio, varía según los productos o la actividad.

\section{Exportación y tránsito}

Pieles de cualquier tipo sin curtir.

Lanas en vellón o por esquilar, tejidos sin terminar de este material e hilazas de lana y estambre.

Mercancias como: aceite, miel, higos, pasas, almendras, palmito y junco y artículos de ambos materiales, cera, lino y cáñamo sin tejer, alheña, azsor, sebo, pez, alquitrán, roldón, zumaque, tanino, sal de compás, quesería, manteca, tocino, manteca de cerdo, jabón, sosa, greda, plata viva, cobre y latón y objetos de estos materiales, acero, hierro, estaño, plomo y cerámica.

Pieles curtidas o preparadas para peleteria, esparto, plata en pan o materia y piezas antiguas de la misma.

Tintes como: grana, rubia, pastel, gualda, orchilla, fustete, alumbre, agalla, tártaro, orchicana; además la ceniza y especias como: pimienta, jengibre, azafrán, comino, matalahuva, clavo, canela. Se incluye además el pescado salado: congrio, arenques, arencada, etc. Este capitulo los primeros años 1404-1405, grava también las armas nuevas: corazas, bacinetes, peces, espadas, cuchillos, lanzas y dardos. 


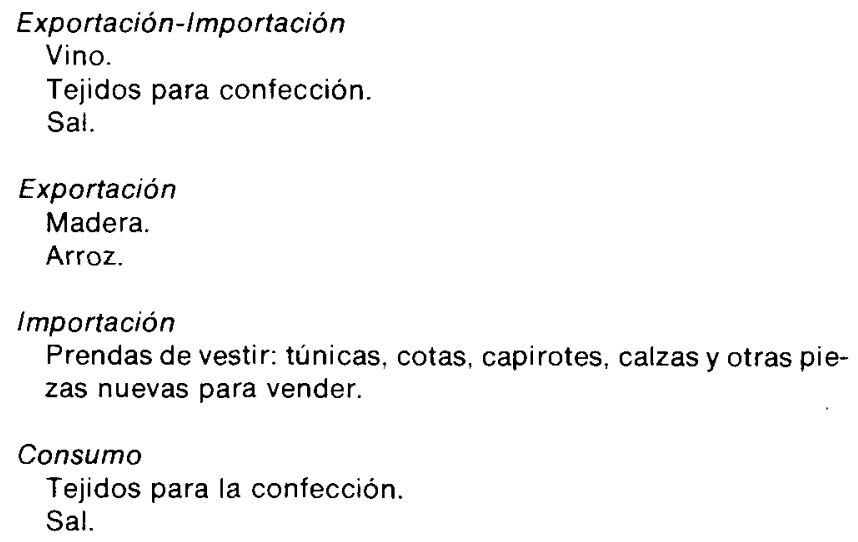

\section{CONCLUSIONES}

Según lo expuesto podemos concluir que el tall del reino, o el impuesto que recae sobre el corte de ciertos tejidos y sobre su importación y exportación, tiene su origen en las Cortes de 1362, donde surge con carácter extraordinario. Carácter que mantendrá con intermitencias hasta 1404. En esa fecha adquiere de hecho, que no de derecho, el rango de ordinario y se mantendrá con ligeras variantes durante toda la época foral.

Se le puede incluir entre los impuestos ordinarios e indirectos de la institución. Su volumen de ingresos en sus tres capitulos: Valencia, Orihuela y el resto del reino es el más importante en el conjunto de los mismos. Su recaudación a través de arrendamiento supone la división del territorio en términos más pequeños, quarters y filloles, que faciliten la gestión de los encargados del cobro.

Los contratos realizados entre los diputados, como máximos representantes de la Generalidad, y los compradores, nos permiten apuntar las cláusulas comunes que rigen las generalidades, asi como los elementos del impuesto que nos ocupa. El trabajo, pues, presenta un doble interés: ser una primera aproximación al mundo fiscal de la Generalidad Valenciana medieval y un eslabón más, que, finalmente, permitirá la clarificación y clasificación del sistema tributario medieval valenciano. 


\section{NOTAS}

(1) PEREZ BUSTAMANTE, Rogelio, Sociedad, economia, fiscalidad y gobierno en las Asturias de Santillana (s. XIII-XV), Santander, Estudio, 1979, pp. 189-192.

(2) El esquema se encuentra en el artículo: «Alzira y la campaña de Pedro IV en Cerdeña (1354)", publicado en Quaderns de Sueca III, 1982, p. 72, y realizado en colaboración con Mercedes GALLENT MARCO.

(3) El estudio de los origenes, composición, medios y atribuciones hasta su consolidación en 1418 es el objeto de nuestra tesis. MARTINEZ ALOY, José, La Diputación de la Generalidad del reino de Valencia, Valencia, 1930, es hasta la fecha el estudio conjunto más importante realizado sobre la misma.

(4) CAMARENA MAHIQUES, José, "Función económica del 'General de regne de Valencia' en el siglo XV", en Anuario de Historia del Derecho Español, t. XXV (1955), pp. 529-542.

(5) Este concepto, empleado por dos titulares al mismo tiempo: la Generalidad y los municipios, tiene elementos que los diversifican, como señala DOÑATE SEBASTIA, José M.", «Sobre el General del tall del drap, o 'del quarter' ", en Boletin de la Sociedad Castellonense de Cultura, t. XLVII, 1971, p. 72.

(6) Vid. mi artículo bases municipales de un impuesto general: "Las Cortes de Valencia de 1329", en Saitabi, XXXIII, 1983, pp. 85-95.

(7) A.R.V., Protocolo núm. 2641, 2642.

(8) La que establece la pena que corresponde al arrendador o recaudador que recauda generalidad de lo que no deba o más de lo señalado.

(9) El riesgo o la fortuna que corra la generalidad queda a cargo del arrendador. Esta cláusula en 1404 sufrirá una puntualización.

(10) El montante de las pujas, gratificaciones, precio de las generalidades de 1404 a 1417 quedan recogidos en el capítulo IV, apartado II, de nuestra tesis dedicado a la organización financiera de la Generalidad.

(11) Sólo han sido localizados algunos datos sobre este punto en A.R.V., Generalidad 4930 (bis). Pero no constan las cantidades ofrecidas por el mismo.

(12) Se especifica en nota los lugares que componen los quarters alicantinos. Del resto sólo se da el primer topónimo, aunque plasmados en un mapa abarcan las provincias actuales de Castellón y Valencia.

(13) A.R.V., Generalidad 596 f. 55-55v "vila, loch e valls de Cocentayna, Planes, Perpunxen e Margarita de totes lurs alqueries termes e pertinencies; e de les viles e lochs e valls de Alcoy, de Sixona, de Penaguila, de Castella ab sa foya, de Tibi, de lbi, de Seta e d'altres lochs d'aquella comarqua ab totes lurs alqueries termes e pertinencies; e de la vila de Alacant, e del loch de Nonpot an tots altres lochs e alqueries de les termes dels dits vila e loch. E mes avant dels lochs d'Elig, e de Crivillen, e de les vaylis e loch d'Ecla, e de Novella, de Azp, de Xinosa, de Monnover e de Petrer ab totes lurs alqueries termes e pertinencies.

(14) Idem, f. 40-44 "Gandia, e dels lochs de Palma, de Vilalonga, de Forna, de Galinera, de Ebbo, de Alcala, de Oliva, e de Rebollet ab totes lurs alqueries, lochs, termens, pertenecies; e de la vila e lochs e vaylls de Denia, de Xabea, de Ondara, del terme de Calp, de Pego, e de les vaylls de Exaló, d'Alaguar, de Murla, de Pop, de Tarbena, de Garig, de Callosa, de Relleu, de 
Finestrat, de Benidorm, de Vilajoyosa, Godalest. de Confrides, de Castell, d'Altea e Bellaguarda ab totes lurs aiqueries termes e pertenencies". En este caso se incluyen algunos lugares hoy pertenecientes a Valencia.

(15) Idem, f. 24-29 "Ontinyen, e de tota la vall de Albayda qui no sia terme de Xativa, e encara dels lochs de Bocayren, Biar, Banyeres y Agres ab totes lurs alqueries termes e pertinencies". Los hemos especificado porque comprende, como puede observarse, algunos lugares hoy pertenecientes a la provincia de Alicante.

(16) Idem, f. 53-53 vo "Oriola e del loch de Guardamar".

(17) Hemos abreviado el nombre de los capitulos. Se recogen completos en nota 29.

(18) Dado que en las notas 13,14, 15 y 16 se especifican todos los topónimos de 1404 , aqui sólo recogemos los que actualmente son núcleos de población.

(19) Sólo las poblaciones subrayadas pertenecen a la actual provincia de Alicante.

(20) A.R.V. Generalidad 596 (bis), f. 44-47, que recoge copia del arrendamiento del tall del reino para 1405 nos proporciona todos los datos aportados en este apartado.

(21) En 1439 los diputados ante las dudas suscitadas sobre este punto delcaran que todas las telas hechas con lana de cualquier clase quedan sometidas al impuesto.

(22) A partir de 1406 se establece sobre los tejidos de: oro, seda, camelote, hosteda, lana estambre, corrdellate y sarga.

(23) Capítulo que se añade a partir de 1406.

(24) Esa exención se añade en 1406.

(25) Esa exención se añade en 1415.

(26) En 1406 el tipo queda evaluado en 6 dineros y a partir de 1432 en 1 sueldo 9 dineros.

(27) Puntualización introducida en 1439.

(28) Las sanciones contenidas en este punto y el anterior se introdujeron en 1421.

(29) La documentación las agrupa para su arrendamiento en diversos capítulos: $1 .^{\circ}$ treta de cuyram cruu e pelos de blanqueria e de pelliceria cruus; $2^{\circ}$ treta de les lanes squilades o per squilar e de/s draps cruus e de les filaçes de lana e d'estam; $3 .^{\circ}$ treta de la fusta nova per obrare del vi e de la entrada del vi strany; $4 .^{\circ}$ treta de diverses robes a mercaderies; $5 .^{\circ}$ treta de les penes e pells apparellades de pelliceria e del argent per obrar e obrat vell e del spart obrat e per obrar; $6 .{ }^{\circ}$ la generalitat de la sal de tot lo regne de Valencia; $70^{\circ}$ Tayll dels draps de la vila de Oriola e del loch de Guardamar; $8 .^{\circ}$ del drap del tayll de la ciutat de Valencia e del terme antich de aquella; 9. Tall dels draps de totes les ciutats, viles e lochs e alqueries de tot lo regne de Valencia exceptada la ciutat de Valencia e la vila de Oriola e loch de Guardamar; $100^{\circ}$ treta de les tintes e de la speciarya e de les armes noves e del peix salat e de la cenra. Todos ellos se arriendan regularmente desde 1404. En 1432 se añade otro conocido bajo el nombre de la quinqualleria y en 1439 el de la Quema.

(30) Este se suprime a partir de 1409.

\section{ABREVIATURAS}

A.C.A. = Archivo de la Corona de Aragón.

A.C.V. = Archivo de la Catedral de Valencia.

A.H.N. = Archivo Histórico Nacional.

A.M.A. = Archivo Municipal de Alicante.

A.M.E. = Archivo Municipal de Elche.

A.P.L. = Archivo de Prehistoria Levantina.

A.R.V. = Archivo del Reino de Valencia.

C.H.I. = Cuadernos de Historia del Islam.

CODOM = Colección de documentos para la historia de Murcia.

CODOIN AGCA = Colección de documentos inéditos del Archivo General de la Corona de Aragón.

N.A.H. = Noticiario Arqueológico Hispánico.

R.A.H. = Real Academia de la Historia. 